\title{
Article
}

\section{Mass Photometry of Membrane Proteins}

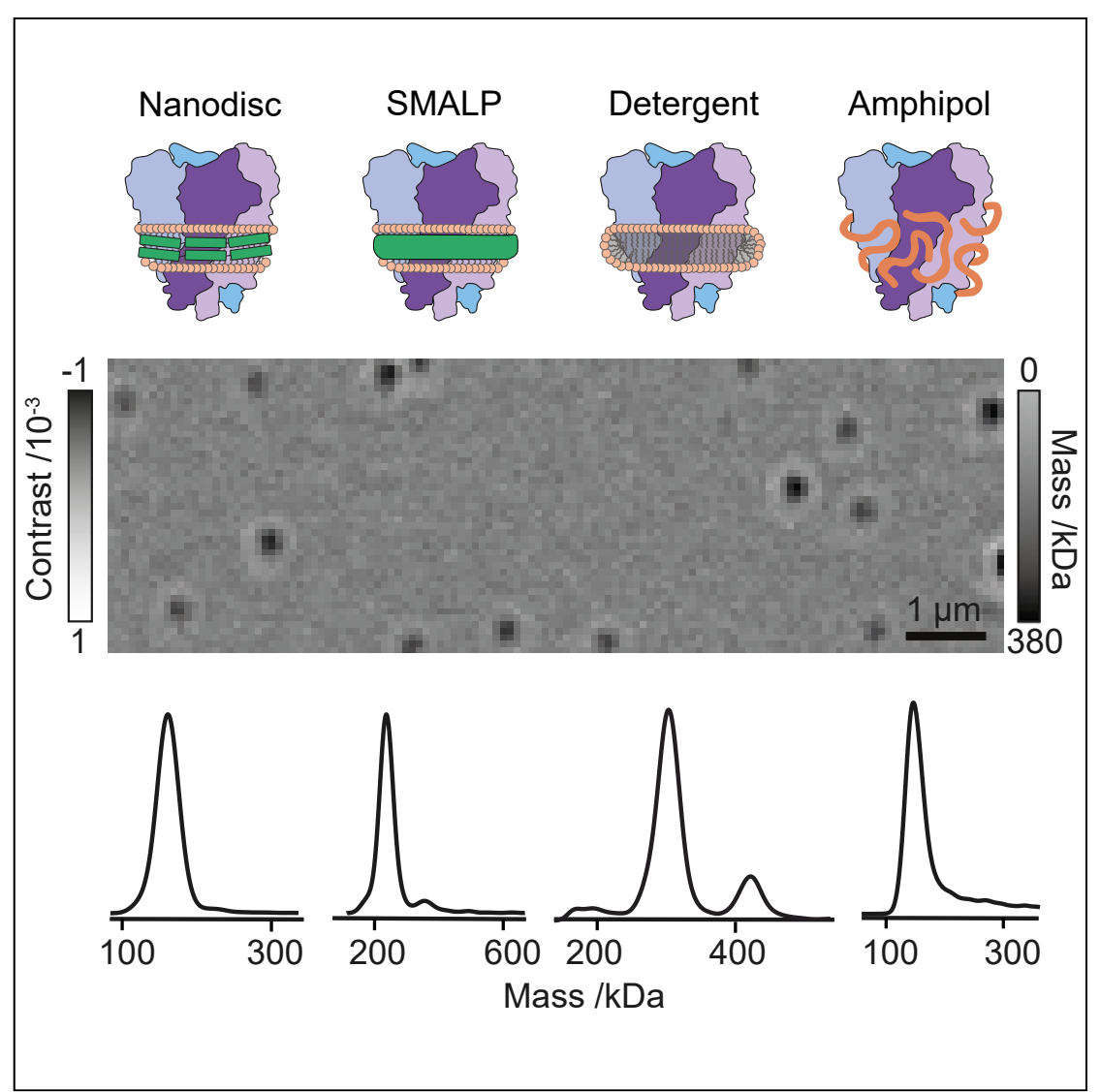

Membrane proteins are important in cell signaling and disease. They are also difficult to study as they require solubilization from lipids by membrane-mimetic systems. We show that mass photometry can facilitate the study of membrane proteins in various mimetic systems. With this method, we can distinguish different oligomeric and functional states of membrane proteins, opening the door for in vitro functional studies, structural characterization, and protein-protein interaction analysis of membrane proteins.
Anna Olerinyova, Adar

Sonn-Segev, Joseph Gault, ..., Roland Riek, Weston B. Struwe, Philipp Kukura

weston.struwe@chem.ox.ac.uk (W.B.S.) philipp.kukura@chem.ox.ac.uk (P.K.)

\section{HIGHLIGHTS}

We introduce a label-free, single molecule approach for membrane-protein characterization

Mass photometry quantifies membrane proteins in different membrane-mimetic systems

MP reveals carrier and protein heterogeneity

It helps distinguish different functional states of membrane proteins 


\title{
Article
}

\section{Mass Photometry of Membrane Proteins}

\author{
Anna Olerinyova, ${ }^{1,6}$ Adar Sonn-Segev, ${ }^{1,6}$ Joseph Gault, ${ }^{1}$ Cédric Eichmann, ${ }^{2}$ Johannes Schimpf, ${ }^{3}$ \\ Adrian H. Kopf, ${ }^{4}$ Lucas S.P. Rudden, ${ }^{5}$ Dzmitry Ashkinadze, ${ }^{2}$ Radoslaw Bomba, ${ }^{2}$ Lukas Frey, ${ }^{2}$ \\ Jason Greenwald, ${ }^{2}$ Matteo T. Degiacomi, ${ }^{5}$ Ralf Steinhilper, ${ }^{2}$ J. Antoinette Killian, ${ }^{4}$ Thorsten Friedrich, ${ }^{3}$ \\ Roland Riek, ${ }^{2}$ Weston B. Struwe, ${ }^{1,7, *}$ and Philipp Kukura ${ }^{1, *}$
}

\section{SUMMARY}

Integral membrane proteins (IMPs) are biologically highly significant but challenging to study because they require maintaining a cellular lipid-like environment. Here, we explore the application of mass photometry (MP) to IMPs and membrane-mimetic systems at the single-particle level. We apply MP to amphipathic vehicles, such as detergents and amphipols, as well as to lipid and native nanodiscs, characterizing the particle size, sample purity, and heterogeneity. Using methods established for cryogenic electron microscopy, we eliminate detergent background, enabling high-resolution studies of membrane-protein structure and interactions. We find evidence that, when extracted from native membranes using native styrenemaleic acid nanodiscs, the potassium channel KcsA is present as a dimer of tetramers-in contrast to results obtained using detergent purification. Finally, using lipid nanodiscs, we show that MP can help distinguish between functional and non-functional nanodisc assemblies, as well as determine the critical factors for lipid nanodisc formation.

\section{INTRODUCTION}

Integral membrane proteins (IMPs) constitute $20 \%$ to $30 \%$ of encoded gene products and have diverse functions, from signaling and transport across membranes to catalysis and mediation of enzymatic reactions. ${ }^{1}$ They represent the majority of small-molecule drug targets, encompassing $\mathrm{G}$ protein-coupled receptors, almost $50 \%$ of which are druggable. ${ }^{2}$ IMPs are generally more complex to study than soluble proteins because their hydrophobic transmembrane region is intrinsically unstable in aqueous solution, requiring astute strategies to solubilize the protein or maintain a native-like lipid environment. As a consequence, only $1.6 \%$ of reported structures in the protein data bank (PDB) correspond to membrane proteins ( 999 IMP out of 61,301 unique protein structures of $<98 \%$ sequence identity). ${ }^{3}$

To overcome the difficulties involved in studying IMP structure and function, a diverse range of membrane-mimetic systems are used as protein carriers varying in their usability and capacity to preserve membrane properties. They include detergent micelles, amphipols, nanodiscs, and lipid particles. ${ }^{4}$ Most often, detergents are used to solubilize and purify IMPs because of their comparative ease of use, making it possible to efficiently extract proteins directly from isolated membranes or intact cells. However, detergent micelles do not necessarily mimic the native lipid bilayer, which can affect the function of proteins stabilized in them. ${ }^{5}$ Amphipols, while more stabilizing than detergents, rely on multiple purification steps, increasing the associated experimental complexity. Nanodisc (ND) mimetic assemblies maintain a lipid

\section{The Bigger Picture}

Membrane proteins are some of the most important biological molecules, carrying out vital functions and being frequent drug targets. Yet, preferring lipid environments and so requiring solubilization, they are challenging to study. Here, we show that mass photometry can characterize the heterogeneity of membrane proteins and the carriers in which they are solubilized. It can also distinguish different functional states of membrane proteins. Our approach thus opens the door to more comprehensive studies of function, structure, and interaction of these critical proteins in their native membrane environment at the singlemolecule level. 
bilayer via annular amphipathic membrane scaffold proteins (MSPs) or styrene-maleic acid (SMA) polymers that encapsulate IMPs in a more native-like environment. SMA lipid particles (SMALPs) and MSP NDs exhibit the improved lateral stability of biological membranes; also, unlike micelles, they do not require excess detergent, and so exhibit a reduced background signal in structural studies. ${ }^{6}$

Membrane-mimetic systems (MMS) are inherently heterogeneous, with varying numbers of lipids and other solubilizing agents. Coupled with the heterogeneity of IMPs, for example, in terms of oligomeric size, studies of IMP structure and function involve challenging and varying combinations of proteins and carriers. This complexity can significantly impact the feasibility, efficacy, and ultimate success of IMP studies. As a result, careful purification and characterization of IMP preparations is essential for both functional and structural studies. Current protein purification and characterization approaches usually rely on a combination of chromatographic techniques, SDS-PAGE, and further analysis by size-exclusion chromatography (SEC), analytical ultracentrifugation, multi-angle light scattering (MALS), negative stain EM, or native mass spectrometry (MS) (where applicable). Though well established, these workflows are time consuming and often challenging. For instance, MALS characterization requires a considerable amount of material (>100 ng) and high-resolution separation by SEC. The success of negative stain EM depends, to some degree, on prior knowledge of likely heterogeneity and sufficient protein size, while native MS of IMPs requires significant expertise and specialized instrumentation. Both of the latter methods necessarily operate under non-native conditions. A method capable of detailed, rapid, and accurate sample characterization in solution could thus dramatically accelerate and improve structural and functional in vitro studies of IMPs.

We have recently introduced mass photometry (MP) and demonstrated its capabilities in terms of determining molecular mass, resolving different oligomeric states, and detecting ligand binding to soluble proteins in a label-free, single-moleculesensitive fashion in solution. ${ }^{7}$ We thus set out to explore the applicability and performance of MP for studying IMPs. Of particular appeal in this context is the universal nature of MP, relying on the detection of changes in the reflectivity of a glass-water interface caused by interference between scattered and reflected light when individual objects bind to the interface ${ }^{8}$ (Figure $1 \mathrm{~A} ;$ Video S1). The reflectivity change upon the molecule landing can then be used to determine the object mass using appropriate calibrants (Figure S1). MP reveals true equilibrium distributions, reports on populations rather than ensembles due to its single-molecule nature and requires minimal sample volumes $(\mu \mathrm{L})$ and concentrations $(<<\mu \mathrm{M})$. Moreover, MP naturally extends toward characterizing structural heterogeneity ${ }^{9}$ and protein-protein interactions, ${ }^{10}$ resulting in a universal platform for studying biomolecules.

\section{RESULTS}

\section{Empty Protein Carriers}

MP is subject to background signatures from nanoscopic empty carriers, because they produce a scattering signal comparable to that of small proteins. To assess the extent to which these could hamper MP measurements, and to help interpret downstream measurements of complex systems, we first studied commonly used MMS in the absence of protein. We began with the detergent lauryl maltose-neopentyl glycol (LMNG) due to its popularity in structural studies, based on enhanced stability of membrane proteins solubilized in this manner. ${ }^{11}$

\footnotetext{
1Physical and Theoretical Chemistry Laboratory, Department of Chemistry, University of Oxford, South Parks Road, Oxford OX1 30Z, UK

2Laboratory of Physical Chemistry, Department of Chemistry and Applied Biosciences, ETH Zürich, Vladimir-Prelog-Weg 1-5/10, 8093 Zürich, Switzerland

Institut für Biochemie,

Albert-Ludwigs-Universität, Alberstraße 21, 79104 Freiburg im Breisgau, Germany

${ }^{4}$ Membrane Biochemistry \& Biophysics, Bijvoet Center for Biomolecular Research, Department of Chemistry, Utrecht University, Padualaan 8, Utrecht $3584 \mathrm{CH}$, the Netherlands

${ }^{5}$ Department of Physics, Durham University, Lower Mountjoy, South Road, Durham DH1 3LE, UK

${ }^{6}$ These authors contributed equally

${ }^{7}$ Lead Contact

${ }^{\star}$ Correspondence:

weston.struwe@chem.ox.ac.uk (W.B.S.), philipp.kukura@chem.ox.ac.uk (P.K.)

https://doi.org/10.1016/j.chempr.2020.11.011
} 

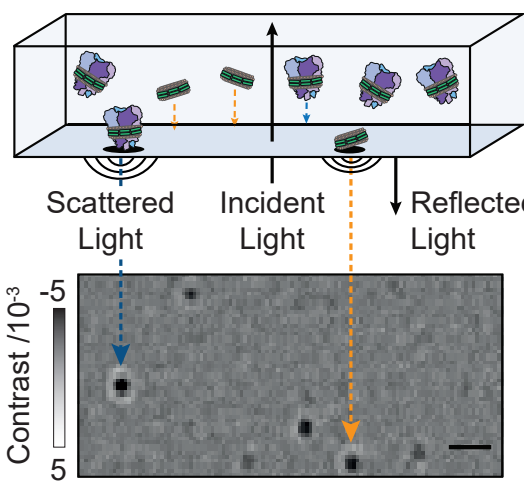

D

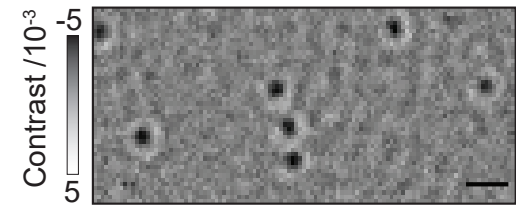

$\mathbf{F}$

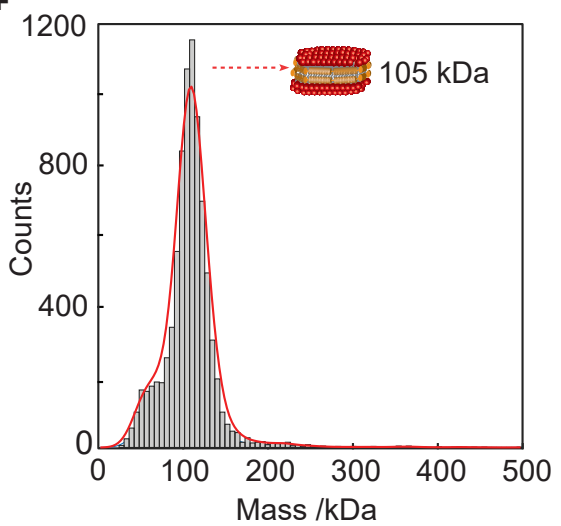

B

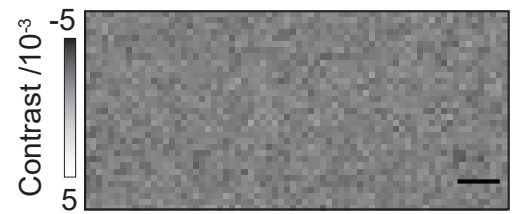

C

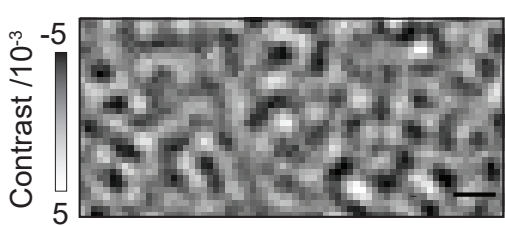

E

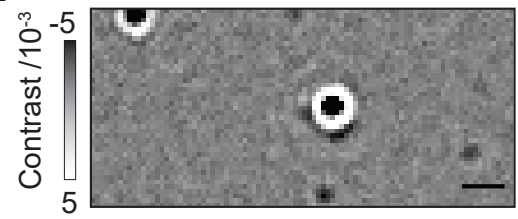

G

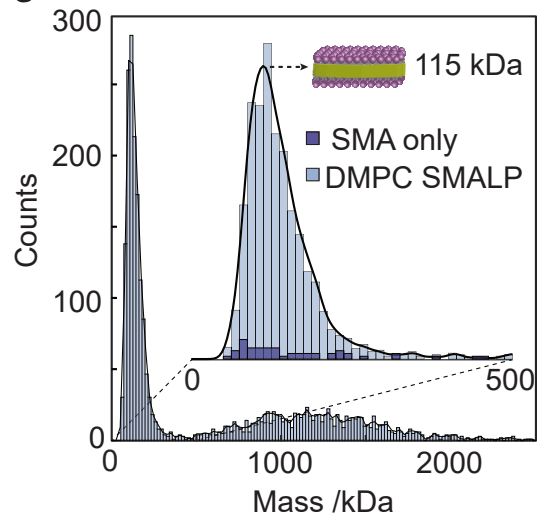

Figure 1. Mass Photometry of Detergent Micelles and Nanodisc Membrane-Protein Carriers (A) Principle of mass photometry. Mass photometry detects light scattering of single biomolecules upon landing on a glass coverslip with each landing event resulting in a change in reflectivity caused by interference of scattered light by the particle and reflected light.

( $B$ and $C$ ) Representative mass photometry images of imaging buffer (B) and LMNG micelles at $2.5 \times$ CMC $(0.0025 \% \mathrm{w} / \mathrm{v})$, respectively.

(D) Empty MSP $\Delta$ H5 DMPC NDs.

(E) Empty DMPC SMALPs at a polymer to lipid ratio of 1:1 (w/w). Scale bars, $1 \mu \mathrm{m}$.

(F) The resulting mass photometry histograms and kernel density estimates for empty MSP NDs.

(G) Corresponding distribution for unoccupied SMALPs (light blue) and SMA polymer aggregates (dark blue). See also Figures S1 and S2 and Videos S1 and S2.

In detergent micelles, using an abundance of detergent above the critical micelle concentration (CMC) aids in solubilizing hydrophobic, transmembrane regions of the IMP, thereby maintaining solubility. To assess the signal generated by detergent micelles in MP, we compared LMNG at $2.5 \times \mathrm{CMC}(0.0025 \% \mathrm{w} / \mathrm{v})$ in buffer $(50 \mathrm{mM}$ MOPS, $20 \mathrm{mM} \mathrm{NaCl}$, pH 7.5) with a buffer blank (Figure 1B). We observed considerable non-specific detergent interactions (Figure 1C; Video S2), due to the effective concentration of LMNG micelles at $2.5 \times \mathrm{CMC}(25 \mu \mathrm{M})^{11}$ being three orders of magnitude larger than the current optimal concentration range for MP (high pM to mid $\mathrm{nM}$ ). This caused surface saturation and rapid binding and unbinding on the 
glass interface, effectively increasing the imaging background, reducing mass resolution, and raising the lower detection limit. Other detergents produced similar signatures at concentrations above the CMC (Figure S2; Videos S3, S4, and S5).

Lipid nanodisc-based systems offer CMC-independent stability, a bilayer-like environment, and potentially lower levels of carrier heterogeneity, as evidenced in our original study. ${ }^{7}$ We therefore imaged MSP $\Delta \mathrm{H} 5$ 1,2-dimyristoyl-sn-glycero-3-phosphocholine (DMPC) lipid nanodiscs (NDs) and SMALPs (SMA polymer to DMPC lipid ratio 1:1 w/w) using MP. NDs and SMALPs differed from LMNG in that distinct, homogeneous particles were detected (Figures $1 \mathrm{D}$ and $1 \mathrm{E}$, respectively). Both unoccupied NDs ( 105 kDa, Figure 1F) and unoccupied SMALPs ( 115 kDa, Figure 1G) produced mostly homogeneous mass distributions. To confirm that we detected lipid-containing SMALPs, as opposed to disassembled polymer aggregates, ${ }^{12}$ we measured SMA polymer at equal concentration as the polymer in unoccupied protein-free SMALPs and found a negligible number of particles (Figure 1G, inset, dark blue histogram). Particle distribution analysis revealed that the standard deviation (SD) of the ND peak fit was $12 \mathrm{kDa}$ or approximately $11.4 \%$. Compared with standard soluble protein SD of approximately $8 \%-10 \%$ of the molecular weight, ${ }^{7}$ NDs and membrane mimetics tend to have broader mass distributions due to variations in the number of constituent molecules present. For SMALPs, the SD of the main peak was even larger at $38 \mathrm{kDa}$, likely arising from varying numbers of lipids and SMA polymers per disc. Unlike MSP NDs, which are limited in size due to the length of the annular belt protein, SMA polymers can, in principle, form discs of unlimited size, but it is unclear what governs the size of assembled SMALPs. ${ }^{13}$ A recent report estimated 140 DMPC lipids per unoccupied SMALP, ${ }^{14}$ which would correspond to approximately $20 \mathrm{kDa}$ of SMA polymer and $95 \mathrm{kDa}$ of DMPC lipids on average as detected by MP. In addition to the main SMALP peak, 40\% of all observed particles were polydisperse and at higher molecular weight, approximately 500-2,000 kDa, indicating some degree of ND oligomerization. In line with this, it has been reported that SMALPs can form oligomeric "rouleaux" stacks, ${ }^{15}$ although this was attributed to a transmission election microscopy (TEM) artifact. This inhomogeneity can also result from an unoptimized SMALP assembly and purification process. MP can, therefore, guide sample production and purification in SMALP experiments.

\section{Detergent and APol Solubilized IMPs}

While imaging background due to unoccupied micelles limits MP performance, we can still distinguish assembled complexes, given sufficient differences in object mass. To illustrate this, we investigated the large E. coli protein $\mathrm{NADH}$ :ubiquinone oxidoreductase (respiratory complex I, $770 \mathrm{kDa}$ including detergent) in LMNG at $1 \times$ CMC (Figure 2A). We could clearly identify assembled protein particles, despite the presence of LMNG, and determine their mass $(770 \mathrm{kDa})$ in excellent agreement with previous studies using analytical ultracentrifugation. ${ }^{16}$

The major remaining limitations were a lack of specificity and detection sensitivity at low mass in the range of micellar size due to interfering detergent background, as well as a loss of mass resolution. Exploiting the slow off-rate of LMNG molecules from IMPs, gradient-based detergent removal (GRaDeR) has shown great potential to effectively remove empty micelles, while leaving membrane proteins solubilized. ${ }^{17}$ We tested a simple 2,000-fold drop dilution of complex I from $5 \times \mathrm{CMC}$ into buffer without LMNG immediately prior to MP measurement. This dilution greatly reduced the detergent background, improving the measurement resolution and enabling us to resolve the low mass peaks. Importantly, the accuracy of the mass 
A



B

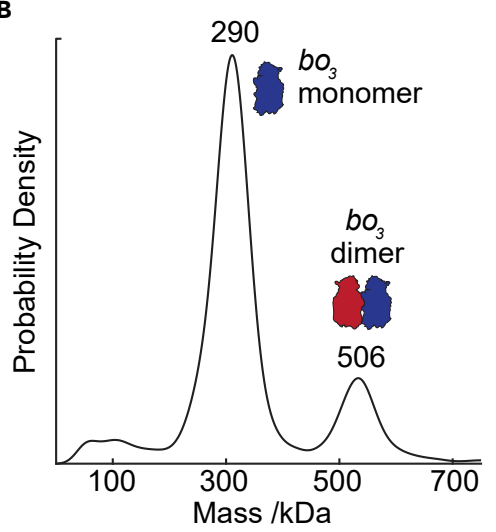

C
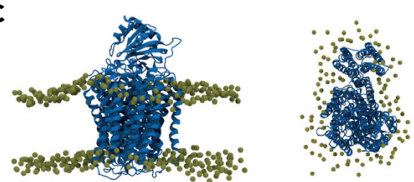

144 kDa monomer + 144 kDa LMNG
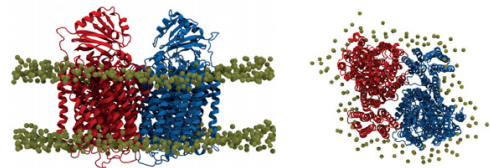

$288 \mathrm{kDa}$ dimer + $221 \mathrm{kDa}$ LMNG

E

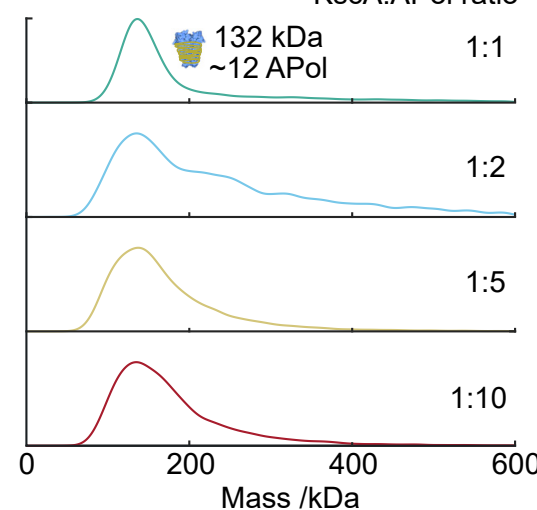

D

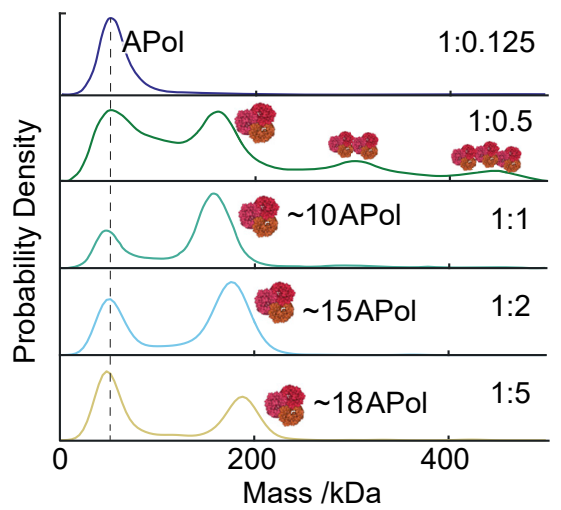

OmpF:APol ratio

Figure 2. MP Analysis of Membrane Proteins with Amphipathic Mimetics

(A) MP of LMNG at $1 \times C M C$ (top) and distributions and typical ratiometric image of respiratory complex I in 1× CMC LMNG (middle) and 2000x LMNG dilution (bottom) after drop dilution. Scale bars, $1 \mu \mathrm{m}$.

(B) Detection of $\mathrm{bo}_{3}$ oxidase monomers and dimers isolated from LMNG detergent micelles by dilution.

(C) Molecular docking and quantitation of bound detergents of $\mathrm{bo}_{3}$ oxidase monomers and dimers ( $D$ and E) (D) OmpF trimers, and (E) KcsA tetramers in amphipols at different protein:APol ratios. KcsA-APol measurements were diluted 200-fold before MP measurement, except for ratio 1:1, which was measured without dilution. See also Figures S3-S7. 
measurement was unaffected by LMNG concentration, showing that IMPs can be studied in the presence of LMNG by simply diluting out excess micelles.

We repeated this drop-dilution approach on the E. coli bo 3 oxidase (144 kDa monomer), where the presence of $\mathrm{bo}_{3}$ dimers in vitro is debated, with some reports declaring the complete absence of dimers in detergent, ${ }^{18}$ and others affirming their existence (although this occurred at the limit of detection, preventing further analysis). ${ }^{19}$ We found monomers and dimers at approximately $80 \%$ and $20 \%$ relative abundances, respectively, (Figure 2B). Here, the measured monomer mass of 290 kDa suggests approximately 146 LMNG detergent molecules (146 kDa) bound to $b_{3}$, making the simplifying assumption of a similar contrast-to-mass conversion for LMNG to that of protein. Similarly, the measured dimer mass (506 kDa) corresponds to approximately 218 LMNG molecules and a $288 \mathrm{kDa}$ protein contribution. In the aforementioned study affirming the dimers' existence, ${ }^{19} \mathrm{bo}_{3}$ dimers were suggested to be non-specific artifacts arising from detergent solubilization. We cannot exclude the possibility that dimer formation is caused by drop dilution from a high LMNG environment, although we did not observe dissociation or significant changes in the relative amount of $\mathrm{bo}_{3}$ dimers at low concentrations over the course of $360 \mathrm{~min}$ (Figure S3). Furthermore, SEC-MALS analysis of $\mathrm{bo}_{3}$ at higher concentration in $0.003 \%$ LMNG (>CMC) was decidedly consistent with our MP analysis-both in terms of molar mass and the relative abundance of monomers and dimers-supporting the occurrence of $\mathrm{bo}_{3}$ dimers in vitro (Figure S4). Specifically, the conjugate bo $_{3}$-LMNG micelle molar mass was $293 \mathrm{kDa}$ (monomer) and $464 \mathrm{kDa}$ (dimer) in 72\% and $28 \%$ relative abundance, respectively, in good agreement with our results.

To test the feasibility of dimer occurrence, we further explored the $\mathrm{bo}_{3}$ dimer structure using protein-protein docking in conjunction with molecular dynamics. ${ }^{20} \mathrm{We}$ simulated the monomer (PDB: 1FFT) ${ }^{21}$ in a 1-palmitoyl-2-oleoyl-glycero-3-phosphocholine (POPC) bilayer for $60 \mathrm{~ns}$, and used this equilibrated structure to generate a pool of possible dimeric arrangements (best candidate is shown in Figure 2C). We then calculated the transmembrane solvent-accessible surface area (SASA) of monomeric $\mathrm{bo}_{3}\left(21,800 \AA^{2}\right)$. Given the mass of LMNG determined by MP (146 kDa), the ratio of SASA per $\mathrm{kDa}$ of detergent is $151.4 \AA^{2} \mathrm{kDa}^{-1}$, which would amount to the presence of 144 lipids. Assuming this ratio is constant between monomeric and dimeric $\mathrm{bo}_{3}$, we derived the mass of LMNG bound to the dimer using, as a consensus value, the mean of dimeric model transmembrane SASA $\left(33,500 \AA^{2}\right)$. We obtained an LMNG mass of $221 \pm 3 \mathrm{kDa}$, a prediction remarkably close to the experimental value of $218 \mathrm{kDa}$. These data support the presence of the $b_{3}$ oxidase dimer in vitro (Figure $2 \mathrm{C}$ ), and illustrate the accuracy of MP in quantifying not only the mass of the polypeptide but also the detergent mass of solubilized membrane proteins.

Amphipols (APols) represent an alternative to detergents for amphipathic IMP solubilization. APols are small polymers $(\sim 4.3 \mathrm{kDa})$ that function similar to detergents, adsorbing at hydrophobic patches on IMPs. However, APols are more stabilizing than detergents and excess APol is not necessarily required to solubilize IMPs, meaning background signals from free APol can be minimized for structural and biophysical methods. ${ }^{22}$ Nevertheless, their use requires an initial detergent solubilization step, adding experimental complexity compared with detergent solubilization alone. MP observations of APols alone were similar to observations of detergent micelles (Video S6).

To examine the applicability of MP to APols, we chose the E. coli outer membrane protein $\mathrm{F}(\mathrm{OmpF})$, which has previously been studied by small-angle neutron 
scattering (SANS) in APols. ${ }^{23}$ OmpF assembles into trimers of $\beta$-barrel monomers and functions as a small-molecule pore with an overall mass of $111 \mathrm{kDa}$ (Figure S5). ${ }^{24}$ MP revealed a ratio-dependent assembly of OmpF trimers solubilized in A8-35 APols from 1:0.125 to 1:5 OmpF to APol ratios (Figure 2D). At or above 1:1 OmpF to APol, MP yielded single peaks of OmpF trimers, suggesting predominantly homogeneous samples. As we increased the APol concentration, we observed a clear mass increase corresponding to larger numbers (approximately 10-18) of bound APols. At sub-stoichiometric ratios, however, we found evidence for dimers and trimers of OmpF oligomers (1:0.5), consistent with published SANS results. ${ }^{23}$ These results suggest an onset of aggregation correlated with loss of APol from OmpFAPol trimers, leading to hydrophobic protein-protein interactions of OmpF trimers. At the extreme 1:0.125 (OmpF:APol) ratio, we could no longer detect any solubilized protein, likely due to large-scale aggregation. We also observed a notable decrease in recovery at a 1:0.25 ratio compared with 1:0.125, but this is likely due to experimental error. Importantly, loss of OmpF during exchange from detergent to amphipols was minor: from 1:0.5 to 1:5 protein:APol ratio based on UV-vis measurements (Supplemental Information, Figure S6). By contrast, a similar titration for the Streptomyces lividans potassium channel, KcsA, did not reveal signs of further oligomerization (Figure 2E). Instead, we observed loss of KcsA during transfer from detergent to APol below 1:2 protein to APol ratios, as observed by UV-vis measurements, which can be rationalized by partial disassembly for KcsA tetramers and protein aggregation (Figure S7).

\section{Native Nanodiscs}

SMA polymers have attracted considerable interest because they can spontaneously solubilize lipid membranes without the need for detergent (Figure 3A). ${ }^{25}$ In short, SMA polymers solubilize IMPs directly from their native environment, retaining natural lipids that may be important for protein stability, assembly, function, and interactions. The heterogeneity inherent in native membranes, i.e., lipid populations, dynamics, oligomerization, and proximity of different proteins, may be better reflected in extracted native NDs. This is opposed to MSP NDs, where IMPs are solubilized in detergent prior to assembly with known lipids and protein belts, and where their limited diameter may restrict applicability to larger complexes.

To explore MP's potential for characterizing native ND preparations and aiding in structural and functional studies, we expressed E. coli KcsA tetramers ( $80 \mathrm{kDa}$ ) and solubilized the membrane using SMA (Xiran 30010). KcsA native NDs were isolated by affinity chromatography and further purified by SEC. Analysis by SDS-PAGE gel (Figure $3 \mathrm{~B}$ ) indicated relatively pure protein preparations, in particular in SEC fractions 13-14. The SEC profile exhibited two main peaks (Figure 3C) and MP analysis of individual fractions taken across both distributions revealed a significant variability in sample heterogeneity (Figures 3D and S8). Fraction 10 revealed a main peak of $\sim 400 \mathrm{kDa}$, with additional peaks at $\sim 250$ and $600 \mathrm{kDa}$, indicating a variation in sample size, which may, in part, be a result of clustering of native nanodiscs or extraction of higher KcsA oligomers. Successive fractions showed a progressive decrease in the number of species, with the most homogeneous fraction 14 eluting at the apex of the second major SEC peak. Fractions 15 and 16, however, were more heterogeneous with a smaller mass shoulder $(\sim 125 \mathrm{kDa})$ next to the main peak ( $\sim 250 \mathrm{kDa})$, consistent with minor quantities of empty SMALPs.

To better understand the oligomerization and composition of the KcsA native NDs, we analyzed our preparation's lipid-to-protein ratio. After affinity purification of the His-tagged KcsA in native NDs, we found that $\sim 44$ lipids were present 
A

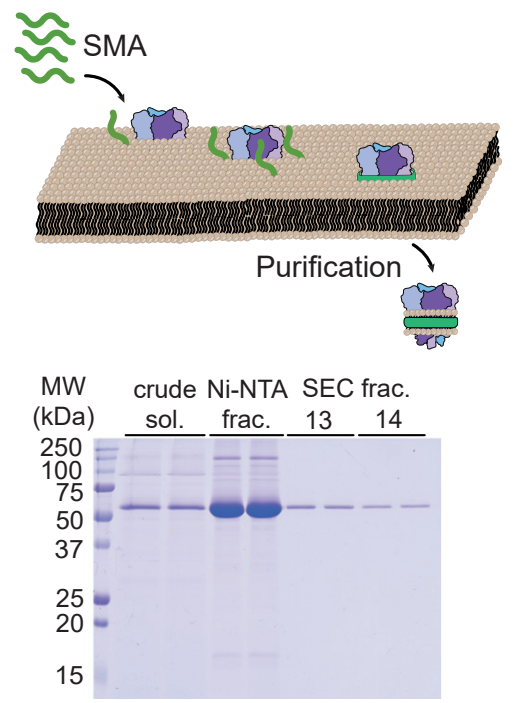

C

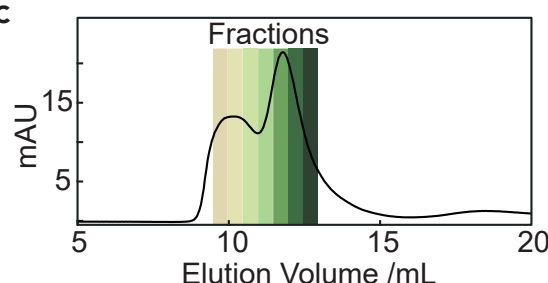

D

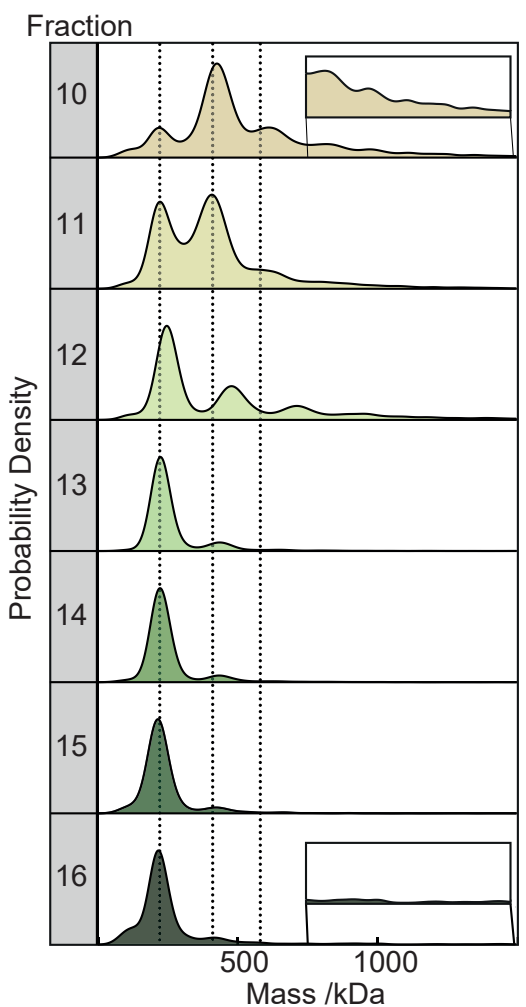

Fraction 16

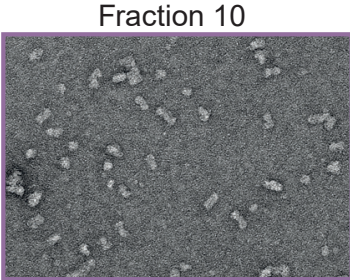

Fraction 11
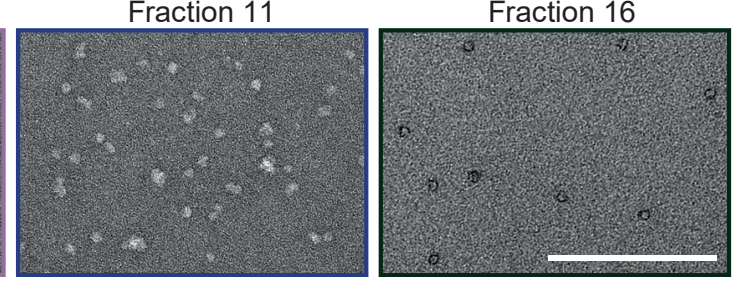

Figure 3. Mass Photometry of KcsA in Nanodiscs

(A) Schematic of native ND formation and extraction of proteins from membranes by SMA.

(B) SDS-PAGE of KcsA native NDs (Xiran 30010 SMA) during purification.

(C) SEC chromatogram of KcsA native NDs, showing the relative protein absorbance at $280 \mathrm{~nm}$.

(D) MP analysis of SEC fractions 10-16. (E) Negative staining EM of fractions 10, 11, and 16

illustrating variability in KcsA native ND assembly. Scale bar, $200 \mathrm{~nm}$. See also Figures S8 and S9 and Table S1.

per KcsA tetramer (Figure S9). Considering a particle size of $\sim 250 \mathrm{kDa}$, and assuming a polymer contribution of $\sim 30 \mathrm{kDa}^{26}$ the remaining lipid-to-protein ratio would be $\sim 200$ if the nanodiscs consisted of a single tetramer per particle, but $\sim 42$ if the nanodiscs were composed of clusters of protein, i.e., dimers of tetramers (Table S1). Though the SEC-purified fractions contained only a limited amount of material, we were able to estimate a lipid-protein ratio in the range of $\sim 20-50$ from fractions 13 and 14, consistent with KcsA being present as dimers of tetramers, in agreement with signatures of clustering reported previously. ${ }^{27-29}$ Additionally, we qualitatively confirmed the observed transition from heterogeneous to homogeneous sample composition by measuring fractions 10,11 , and 16 with negative stain electron microscopy (Figure 3E), which showed larger species in fractions 10 and 11, and a homogeneous distribution of smaller species in fraction 16. 
A
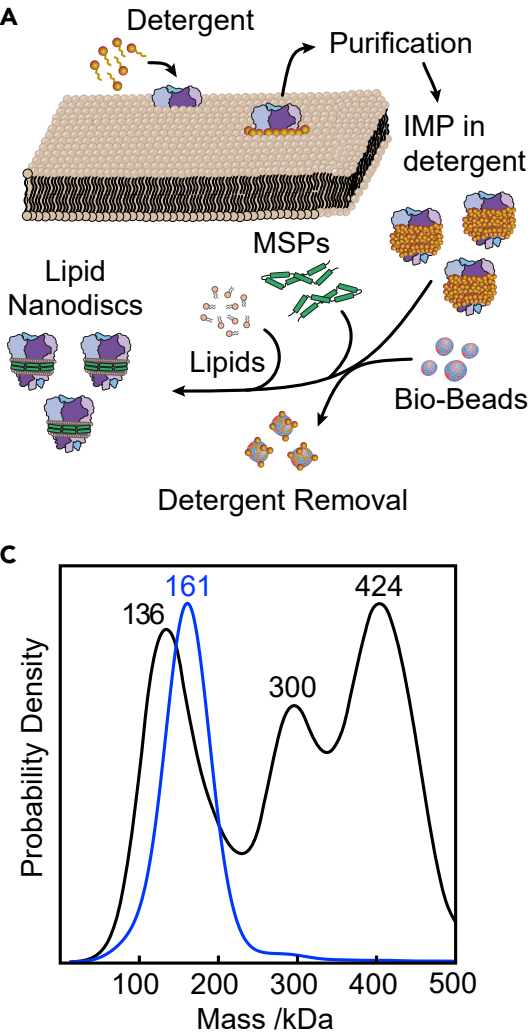

B

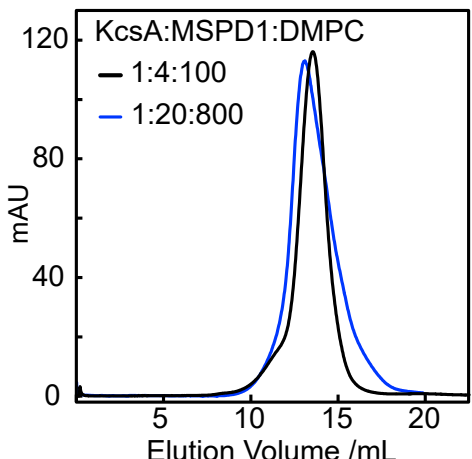

D

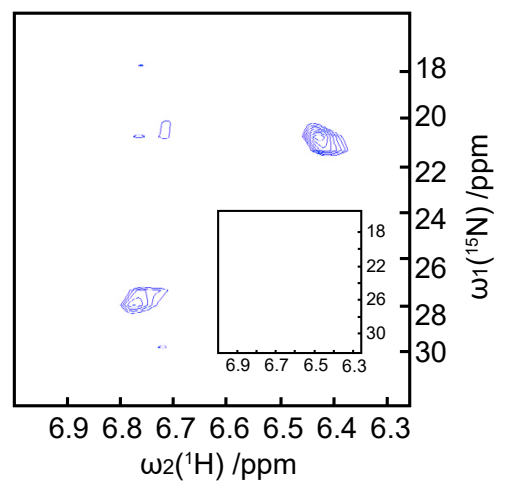

Figure 4. Mass Photometry of KcsA in Lipid Nanodiscs

(A) Schematic showing KcsA solubilization and insertion into lipid nanodiscs.

(B) SEC chromatograms of KcsA MSP NDs assembled at different KcsA:MSP1D1:DMPC ratios.

(C) Corresponding MP analysis.

(D) $2 \mathrm{D}\left[{ }^{15} \mathrm{~N},{ }^{1} \mathrm{H}\right]-\mathrm{HMQC}$ spectra revealing $\mathrm{NH}_{4}{ }^{+}$binding by $\mathrm{KcsA}$ in native NDs prepared in a

1:20:800 KcsA:MSP1D1:DMPC ratio, but no signal was detected from 1:4:100 ratios demonstrating protein inactivity (inset). See also Figures S10 and S11 and Table S2.

\section{Lipid Nanodiscs}

The high resolution of MP also enabled us to screen and optimize the conditions for reconstitution of KcsA from S. lividans into MSP NDs. After selecting the lipid entity and the MSP variant, there are three main variables in the preparation of KcsA embedded nanodiscs: the absolute and relative concentrations of KcsA, MSP1D1, and the lipids (Figure 4A). In the initial screen (condition B in Table S2), a KcsA:MSP1D1:DMPC lipid ratio of 1:4:100 was selected on the basis of an excellent size-exclusion profile (Figure 4B, black). The MP profile clearly indicated three different species with masses of 118,280 , and $391 \mathrm{kDa}$ corresponding, respectively, to empty ND, ND with two KcsA tetramers, and two fused NDs (Figure 4C, black). By contrast, a KcsA:MSP1D1:DMPC lipid ratio of 1:20:800 exhibited a slightly poorer size-exclusion profile (Figure 4B, blue), but a much more homogeneous MP profile (Figure 4C, blue), with a mass of $161 \mathrm{kDa}$ corresponding to a single KcsA tetramer embedded in a nanodisc consisting of 2 MSP1D1 proteins and approximately 25 lipids. The latter preparation exhibited ${ }^{15} \mathrm{NH}_{4}{ }^{+}$ion binding upon addition of $50 \mathrm{mM}^{15} \mathrm{NH}_{4}{ }^{+}$, as revealed by the presence of 2 strong and 2 very weak $\mathrm{NH}_{4}{ }^{+}$cross peaks in a $2 \mathrm{D}\left[{ }^{15} \mathrm{~N},{ }^{1} \mathrm{H}\right]-\mathrm{HMQC} \mathrm{NMR} \mathrm{spec-}$ trum assigned to the 4 ion binding sites in the selectivity filter of KcsA (Figure 4D). By contrast, we could not detect any signatures of ion binding in the former sample preparation shown to be highly heterogeneous and lacking KcsA tetramers by MP, demonstrating the lack of functional KcsA (Figure 4D, inset). 
To further evaluate which of the possible assembly ratios is essential for ensuring assembly and purification of KcsA tetramers, we carried out a screen to determine the optimal IMP to scaffold $(\mathrm{mol} / \mathrm{mol}$ ) ratio with a fixed scaffold:lipid ratio of $1: 40$ at a lipid concentration of $16 \mathrm{mM}$. We monitored sample absorbance at $280 \mathrm{~nm}$, indicative of KcsA content, for the following conditions: the initial assembly mixture, the assembly mixture after overnight incubation, the washing fraction from batch $\mathrm{Ni}^{2+}-\mathrm{NTA}$ chromatography, and the elution fraction of purified KcsA nanodiscs (Figure S10). Characterizing all resulting KcsA MSP ND samples by MP revealed homogeneous samples with a consistent mass around $161 \mathrm{kDa}$ (Figure S11). These results suggest that the IMP:scaffold molar ratio is not crucial for correct sample assembly. Instead, the key parameter controlling the functionality of KcsA embedded in MSP NDs is the scaffold:lipid ratio, contrary to previously published protocols, ${ }^{30}$ highlighting the potential of MP in future nanodisc studies.

\section{DISCUSSION}

These results demonstrate that MP can accurately characterize membrane-protein carriers and IMPs at the single-particle level in solution. Given its universal applicability, speed, and ease of operation, MP is likely to have a significant impact on in vitro studies of IMPs. While many of the results from both empty and filled amphipathic carriers can be obtained with alternative techniques at the ensemble level, none can match the speed (60 s per experiment), low sample requirements (<pmoles), and single-particle sensitivity and resolution of MP. For SMALPs, we showed the dramatic difference in sample information as revealed by SEC compared with MP. Such sub-chromatographic resolution characterization of samples during purification holds great promise to improve and accelerate sample preparation for subsequent structural or functional studies. In the case chosen (Figure 3), for example, fraction 15 would be immediately suitable for subsequent characterization by cryoEM, providing a route to experimentally test whether detergent and native environments are indeed indistinguishable, ${ }^{31}$ although the fact that our SMALPs consist almost exclusively of dimers of tetramers already points toward non-negligible differences between the two. The stark differences between the information content from SEC and MP are further illustrated by our results on KcsA in MSP NDs. Two almost indistinguishable SEC profiles were shown to consist of completely different assemblies, with only one, the one identified by MP, being functional.

Despite MP's advantages, limitations remain: (1) $\mathrm{MP}^{\prime} \mathrm{s}$ concentration range is currently limited to the $<100 \mathrm{nM}$ regime, which represents a challenge for the majority of detergents. This can be addressed by borrowing from already proven approaches for translating single-molecule techniques from the $\mathrm{nM}$ to the $\mu \mathrm{M}$ or even $\mathrm{mM}$ regime, ${ }^{32}$ or surface passivation approaches. (2) The output of MP is not protein specific. Gaining insight on protein versus lipid mass thus requires some a priori knowledge of the protein mass. (3) The masses reported herein are derived from a calibration based on globular polypeptides. Given that lipids have a different polarizability to polypeptides, and one that indeed may also vary depending on their nanoscopic location, such as within a nanodisc compared with contact with solution as in detergent, our mass determination for lipid-polypeptide constructs are unlikely to be highly accurate. This could be improved if well-defined mass standards-consisting of lipids only, or mixtures of lipids and polypeptides were available. As a result, we have no means to strictly evaluate the accuracy of our mass measurements. Nevertheless, we 
have previously found close correlation between MP-measured and predicted mass changes as a consequence of lipid changes alone, ${ }^{7}$ and seen mass accuracy of a polypeptide calibration on the order of $20 \%$ even when compared with DNA, which is structurally completely different to globular proteins, ${ }^{33}$ suggesting that the achievable mass accuracy is reasonable. In future, a more quantitative scale could be provided by obtaining robust standards, or by explicit comparison with native MS measurements, assuming, where possible, that no lipids or weakly associated species are lost during the measurement.

Our results should not, however, be viewed exclusively in the context of comparison with existing sample characterization techniques. Existing techniques are very powerful and have enabled and continue to yield detailed information on sample composition and heterogeneity. Key to MP is the combination of speed and simplicity, as well its broader capabilities, which include detection of small ligand binding, ${ }^{7}$ quantitative evaluation of binding affinities and kinetics in a surface free manner, ${ }^{10,34}$ nucleic acid interactions ${ }^{33}$ and more general capabilities for characterizing sample heterogeneity. ${ }^{9}$ All of these measurements are performed in the same way as the ones presented herein: by adding small volumes $(\mu \mathrm{L})$ of low concentration $(<\mu \mathrm{M})$ unlabeled samples in a buffer of choice to a microscope cover slide. This combination of speed and ease of use with a broad feature palette of additional capabilities will make MP a powerful, universal method to study IMP structure, function, and interactions.

\section{EXPERIMENTAL PROCEDURES}

\section{Resource Availability}

\section{Lead Contact}

Further information and requests for resources and reagents should be directed to and will be fulfilled by the Lead Contact, Weston Struwe (weston.struwe@chem. ox.ac.uk).

\section{Materials Availability}

This study did not generate new unique reagents.

Data and Code Availability

The datasets supporting this study are available through the Oxford Research Archive at https://doi.org/10.5287/bodleian:5zNMEZYOG.

\section{Materials and Methods}

Extended experimental methods and materials can be found in Supplemental Information. Each MP measurement was performed on glass coverslips and recorded on mass photometers (OneMP, Refeyn Ltd) ${ }^{7}$ for 60-90 s. Each measurement was repeated at least 3 times. The samples were diluted immediately prior to measurements in buffer to 10-50 nM (for exact concentrations, see Table S3). The recorded videos were analyzed using DiscoverMP (Refeyn Ltd, version 2.2.0) to quantify protein binding events, with analysis parameters outlined in Table S4. The molecular weight was obtained by contrast comparison with known mass standard calibrants measured on the same day. The proteins used for MP measurements were overexpressed in bacterial cell lines, extracted with either detergent micelles or SMA copolymer and purified using NTA-Ni ${ }^{2+}$ and SEC. Detergent-purified IMPs were either measured directly or exchanged into lipid nanodiscs and amphipols using detergent-absorbent bio-beads. SEC-MALS analysis was performed on an Agilent 1200 HPLC system (Wyatt Technology) and light scattering data were analyzed as a protein conjugate using the Astra $\mathrm{V}$ software. For negative staining electron 
microscopy, grids were glow discharged and $10 \mu \mathrm{L}$ of sample was applied for 2 min, blotted, stained with $2 \%$ uranyl acetate and allowed to air dry. Images were acquired on a $120 \mathrm{kV}$ Tecnai 12 (ThermoFisher) TEM equipped with a OneView digital camera (Gatan). 2D NMR spectroscopy [15N, 1H]-HMOC experiments with $\mathrm{H}_{2} \mathrm{O} /{ }^{15} \mathrm{NH}_{4}{ }^{+}$defocusing and selective excitation burp pulses were all collected at $305 \mathrm{~K}$ on a Bruker $700 \mathrm{MHz}$ Avance III spectrometer equipped with a triple resonance cryoprobe. Molecular dynamics (MD) simulations of $\mathrm{bo}_{3}$ ubiquinol oxidase (PDB: 1FFT) were carried out in a lipid bilayer. ${ }^{21}$ All simulations were run in the NPT ensemble. The system was simulated for $60 \mathrm{~ns}$, where the first $50 \mathrm{~ns}$ were excluded as part of the equilibration, and the last $10 \mathrm{~ns}$ used for production. The prediction of a possible $\mathrm{bo}_{3}$ dimeric arrangement was carried out using protein-protein docking software. The last $10 \mathrm{~ns}$ of the MD simulation were extracted to generate a spatial and temporal influence density (STID) map of $\mathrm{bo}_{3}$ representative of its local dynamics in a lipid bilayer. The docking procedure evaluated 36,000 docked poses. The dimer shown in Figure 2C is a representative model of the ensemble. For native MS, the mass spectra were recorded on a prototype Orbitrap $\mathrm{Q}$ Exactive ultra-high mass range (UHMR) mass spectrometer (Thermo Fisher), equipped with a nanospray source. OmpF, prepared in $100 \mathrm{mM}$ ammonium acetate at $2 \times \mathrm{CMC}$ octyl $\beta$-D-glucopyranoside detergent was transferred into the mass spectrometer using glass capillaries. The mass spectrometer was operated in positive ion polarity, ion optics transmission was set to high $\mathrm{m} /$ $z$, and detector mode to low $\mathrm{m} / \mathrm{z}$.

\section{SUPPLEMENTAL INFORMATION}

Supplemental Information can be found online at https://doi.org/10.1016/j.chempr. 2020.11.011.

\section{ACKNOWLEDGMENTS}

P.K. is supported by an ERC Consolidator grant (PHOTOMASS 819593). A.O. is supported by an EPSRC DTC Studentship. J. Gault is supported by a Junior Research Fellowship at the Queen's College, Oxford. M.T.D. is supported the EPSRC fellowship EP/P016499/1. W.B.S. was supported by Refeyn Ltd. The work in the T.F. laboratory was supported by the Deutsche Forschungsgemeinschaft (DFG) grant 278002225/RTG 2202. We thank N.G. Housden and C. Kleanthous for providing OmpF protein. We thank Catherine Lichten for feedback.

\section{AUTHOR CONTRIBUTIONS}

Concept, W.B.S. and P.K.; Methodology, A.O., A.S.S., J. Gault, W.B.S., P.K.; Investigation, A.O., A.S.S., J. Greenwald, D.A., R.B., C.E., J.S., A.H.K., L.S.P.R., M.T.D., and L.F.; Formal Analysis, A.O. and A.S.S.; Writing - Original Draft, W.B.S., and P.K.; Writing - Review \& Editing, all authors; Visualization, A.O., A.S.S., W.B.S., and P.K.; Supervision: J. Gault, J.A.K., T.F., A.S.S., R.R., W.B.S., and P.K.

\section{DECLARATION OF INTERESTS}

P.K. is a director, founder, and shareholder in Refeyn Ltd. W.B.S. is a shareholder and consultant to Refeyn Ltd. All other authors declare no conflict of interest.

Received: May 14, 2020

Revised: August 20, 2020

Accepted: November 11, 2020

Published: December 4, 2020 


\section{REFERENCES}

1. Fagerberg, L., Jonasson, K., Von Heijne, G., Uhlén, M., and Berglund, L. (2010). Prediction of the human membrane proteome. Proteomics 10, 1141-1149.

2. Hauser, A.S., Attwood, M.M., Rask-Andersen, M., Schiöth, H.B., and Gloriam, D.E. (2017) Trends in GPCR drug discovery: new agents, targets and indications. Nat. Rev. Drug Discov. 16, 829-842.

3. Wang, G., and Dunbrack, R.L. (2003). PISCES: a protein sequence culling server. Bioinformatics 19, 1589-1591.

4. Catoire, L.J., Warnet, X.L., and Warschawski, D.E. (2014). Micelles, bicelles, amphipols, nanodiscs, iposomes, or intact cells: the hitchhiker's guide to the study of membrane proteins by NMR. In Membrane Proteins Production for Structural Analysis, I. MusVeteau, ed. (Springer), pp. 315-345.

5. Chung, K.Y., Kim, T.H., Manglik, A., Alvares, R., Kobilka, B.K., and Prosser, R.S. (2012). Role of detergents in conformational exchange of a $\mathrm{G}$ protein-coupled receptor. J. Biol. Chem. 287, 36305-36311.

6. Gewering, T., Januliene, D., Ries, A.B., and Moeller, A. (2018). Know your detergents: A case study on detergent background in negative stain electron microscopy. J. Struct. Biol. 203, 242-246.

7. Young, G., Hundt, N., Cole, D., Fineberg, A. Andrecka, J., Tyler, A., Olerinyova, A., Ansari, A., Marklund, E.G., Collier, M.P., et al. (2018). Quantitative mass imaging of single biological macromolecules. Science 360, 423-427.

8. Cole, D., Young, G., Weigel, A., Sebesta, A., and Kukura, P. (2017). Label-Free singlemolecule imaging with numerical-apertureshaped interferometric scattering microscopy. ACS Photonics 4, 211-216.

9. Sonn-Segev, A., Belacic, K., Bodrug, T., Young, G., VanderLinden, R.T., Schulman, B.A., Schimpf, J., Friedrich, T., Dip, P.V., Schwartz, T.U., et al. (2020). Quantifying the heterogeneity of macromolecular machines by mass photometry. Nat. Commun. 11, 1772.

10. Soltermann, F., Foley, E.D.B., Pagnoni, V., Galpin, M., Benesch, J.L.P., Kukura, P., and Struwe, W.B. (2020). Quantifying proteinprotein interactions by molecular counting with mass photometry. Angew. Chem. Int. Ed. Engl. 59, 10774-10779.

11. Breyton, C., Javed, W., Vermot, A., Arnaud, C.A., Hajjar, C., Dupuy, J., Petit-Hartlein, I., Le Roy, A., Martel, A., Thépaut, M., et al. (2019). Assemblies of lauryl maltose neopentyl glycol (LMNG) and LMNG-solubilized membrane proteins. Biochim Biophys Acta Biomembr. 1861, 939-957.

12. Scheidelaar, S., Koorengevel, M.C., van Walree, C.A., Dominguez, J.J., Dörr, J.M., and Killian, J.A. (2016). Effect of polymer composition and $\mathrm{pH}$ on membrane solubilization by styrene-maleic acid copolymers. Biophys. J. 111, 1974-1986.

13. Dörr, J.M., Scheidelaar, S., Koorengevel, M.C., Dominguez, J.J., Schäfer, M., van Walree, C.A., and Killian, J.A. (2016). The styrene-maleic acid copolymer: a versatile tool in membrane research. Eur. Biophys. J. 45, 3-21.

14. Jamshad, M., Grimard, V., Idini, I., Knowles, T.J., Dowle, M.R., Schofield, N., Sridhar, P., Lin, Y.P., Finka, R., Wheatley, M., et al. (2015). Structural analysis of a nanoparticle containing a lipid bilayer used for detergent-free extraction of membrane proteins. Nano Res 8, 774-789.

15. Dominguez Pardo, J.J., Dörr, J.M., Renne, M.F., Ould-Braham, T., Koorengevel, M.C., van Steenbergen, M.J., and Killian, J.A. (2017). Thermotropic properties of phosphatidylcholine nanodiscs bounded by styrene-maleic acid copolymers. Chem. Phys. Lipids 208, 58-64.

16. Böttcher, B., Scheide, D., Hesterberg, M., Nagel-Steger, L., and Friedrich, T. (2002). A novel, enzymatically active conformation of the Escherichia coli NADH:ubiquinone oxidoreductase (complex I). J. Biol. Chem. 277, 17970-17977.

17. Hauer, F., Gerle, C., Fischer, N., Oshima, A., Shinzawa-Itoh, K., Shimada, S., Yokoyama, K. Fujiyoshi, Y., and Stark, H. (2015). GraDeR: membrane protein complex preparation for single-particle cryo-EM. Structure 23, $1769-$ 1775.

18. Musatov, A., Ortega-Lopez, J., Demeler, B. Osborne, J.P., Gennis, R.B., and Robinson, N.C. (1999). Detergent-solubilized Escherichia coli cytochrome bo3 ubiquinol oxidase: a monomeric, not a dimeric complex. FEBS Lett. 457, 153-156.

19. Palombo, I., and Daley, D.O. (2012). Heme incorporation into the cytochrome bo3 occurs at a late stage of assembly. FEBS Lett 586, 4197-4202.

20. Rudden, L.S.P., and Degiacomi, M.T. (2019) Protein docking using a single representation for protein surface, electrostatics, and local dynamics. J. Chem. Theor. Comput. 15, 51355143.

21. Abramson, J., Riistama, S., Larsson, G., Jasaitis, A., Svensson-Ek, M., Laakkonen, L., Puustinen, A., Iwata, S., and Wikström, M. (2000). The structure of the ubiquinol oxidase from Escherichia coli and its ubiquinone binding site. Nat. Struct. Biol. 7, 910-917.

22. Zoonens, M., and Popot, J.L. (2014). Amphipols for each season. J. Membr. Biol. 247, 759-796.

23. Arunmanee, W., Heenan, R.K., and Lakey, J.H. (2018). Determining the amphipol distribution within membrane-protein fibre samples using small-angle neutron scattering. Acta Crystallogr. D Struct. Biol. 74, 1192-1199.
24. Gault, J., Donlan, J.A.C., Liko, I., Hopper, J.T.S., Gupta, K., Housden, N.G., Struwe, W.B., Marty, M.T., Mize, T., Bechara, C., et al. (2016). Highresolution mass spectrometry of small molecules bound to membrane proteins. Nat. Methods 13, 333-336.

25. Dörr, J.M., Koorengevel, M.C., Schäfer, M., Prokofyev, A.V., Scheidelaar, S., Van Der Cruijsen, E.A.W., Dafforn, T.R., Baldus, M., and Killian, J.A. (2014). Detergent-free isolation, characterization, and functional reconstitution of a tetrameric K+ channel: the power of native nanodiscs. Proc. Natl. Acad. Sci. USA 111, 18607-18612.

26. Stroud, Z., Hall, S.C.L., and Dafforn, T.R. (2018) Purification of membrane proteins free from conventional detergents: SMA, new polymers, new opportunities and new insights. Methods 147, 106-117.

27. Visscher, K.M., Medeiros-Silva, J., Mance, D., Rodrigues, J.P.G.L.M., Daniëls, M., Bonvin, A.M.J.J., Baldus, M., and Weingarth, M. (2017) Supramolecular organization and functional implications of $\mathrm{K}+$ channel clusters in membranes. Angew. Chem. Int. Ed. Engl. 56, 13222-13227.

28. Giudici, A.M., Molina, M.L., Ayala, J.L., Montoya, E., Renart, M.L., Fernández, A.M., Encinar, J.A., Ferrer-Montiel, A.V., Poveda, J.A., and González-Ros, J.M. (2013). Detergentlabile, supramolecular assemblies of KcsA: relative abundance and interactions involved. Biochim Biophys Acta 1828, 193-200.

29. Molina, M.L., Barrera, F.N., Fernández, A.M., Poveda, J.A., Renart, M.L., Encinar, J.A. Riquelme, G., and González-Ros, J.M. (2006). Clustering and coupled gating modulate the activity in KcsA, a potassium channel model. J. Biol. Chem. 281, 18837-18848.

30. Hagn, F., Nasr, M.L., and Wagner, G. (2018). Assembly of phospholipid nanodiscs of controlled size for structural studies of membrane proteins by NMR. Nat. Protoc. 13, 79-98.

31. Matthies, D., Bae, C., Toombes, G.E.S., Fox, T., Bartesaghi, A., Subramaniam, S., and Swartz, K.J. (2018). Single-particle cryo-EM structure of a voltage-activated potassium channel in lipid nanodiscs. eLife 7, e37558.

32. Zijlstra, N., Dingfelder, F., Wunderlich, B. Zosel, F., Benke, S., Nettels, D., and Schuler, B. (2017). Rapid microfluidic dilution for singlemolecule spectroscopy of low-affinity biomolecular complexes. Angew. Chem. Int. Ed. Engl. 56, 7126-7129.

33. Li, Y., Struwe, W.B., and Kukura, P. (2020). Single molecule mass photometry of nucleic acids. Nucleic Acids Res 48, e97.

34. Wu, D., and Piszczek, G. (2020). Measuring the affinity of protein-protein interactions on a single-molecule level by mass photometry. Anal. Biochem. 592, 113575. 